\title{
Narrativa
}

\section{L'esorbitante, il sovversivo, l'osceno: riflessioni sul trattamento del corpo nella recente narrativa italiana}

\section{Matteo Ottaviano}

\section{CpenEdition}

\section{Journals}

Edizione digitale

URL: https://journals.openedition.org/narrativa/366

DOI: $10.4000 /$ narrativa.366

ISSN: 2804-1224

\section{Editore}

Presses universitaires de Paris Nanterre

Edizione cartacea

Data di pubblicazione: 1 décembre 2019

Paginazione: 143-155

ISBN: 978-2-84016-350-3

ISSN: 1166-3243

\section{Notizia bibliografica digitale}

Matteo Ottaviano, «L'esorbitante, il sovversivo, l'osceno: riflessioni sul trattamento del corpo nella recente narrativa italiana», Narrativa [Online], 41 | 2019, online dal 01 novembre 2021, consultato il 08 décembre 2021. URL: http://journals.openedition.org/narrativa/366 ; DOI: https://doi.org/10.4000/ narrativa.366

Narrativa est mise à disposition selon les termes de la Licence Creative Commons Attribution 4.0 International 


\title{
L'esorbitante, il sovversivo, l'osceno: riflessioni sul trattamento del corpo nella recente narrativa italiana
}

\begin{abstract}
RIASSUNTO
La narrativa italiana degli anni Duemila sembra presentare alcuni nuclei tematici comuni che tendono a porre un argine alla dimensione frastagliata e varia della moltitudine di scritture recenti e alla mancanza di scuole riconoscibili e riconosciute. In questo articolo si è deciso di esplorare uno di questi nuclei: il corpo. Lo si è fatto attraversando tre opere molto diverse, per struttura e tematica, di tre autori coevi, Giorgio Vasta, Nicola Lagioia e Giorgio Falco.

\section{RÉSUMÉ}

La littérature italienne des années 2000 semble présenter des noyaux thématiques communs, ce qui tende à circonscrire la dimension découpée et variée de la multitude d'écritures récentes et pallier le manque d'écoles reconnaissables et reconnues. Cet article se concentre sur l'un de ces noyaux thématiques, le corps, en analysant les œuvres de trois auteurs contemporains : Giorgio Vasta, Nicola Lagioia et Giorgio Falco.
\end{abstract}

a mancanza di scuole e correnti riconoscibili non ha impedito alla recente narrativa italiana di produrre nuclei tematici e caratteri stilistici che lasciano intravedere, se non una forte compattezza, almeno una determinata coerenza. Tale coerenza pare configurarsi come una risposta sintomatologica ad alcune richieste sollevate dalla contemporaneità, in particolare la necessità di doversi confrontare con i sistemi di comunicazione di massa e con il riaffiorare di conflitti e di crisi a seguito dell'apparente pacificazione avvenuta tra la fine degli anni Settanta e l'inizio degli anni Novanta ${ }^{1}$. Sotto il profilo stilistico possiamo infatti notare una generale tendenza alla rapidità e al periodo

1. Luperini Romano, La fine del postmoderno, Napoli, Alfredo Guida Editore, 2008, p. 12. 
breve e una predisposizione all'ibridazione con altre forme espressive (inchiesta, canzone, fumetto, cinema, televisione), mentre la lingua talvolta riprende l'italiano neostandard impostosi tra gli anni Ottanta e Novanta e talvolta prova a riprodurre in una chiave iperrealistica il linguaggio orale regionale o gergale ${ }^{2}$. A livello tematico si possono rinvenire tre macrotendenze: l'interesse per la recente storia italiana, in particolare gli anni Settanta e Ottanta avvertiti come fondativi del quadro sociale e politico attuale; il riaffiorare dell'impegno che trascina con sé poetiche realistiche e una volontà testimoniale; la presenza della dimensione fisica dell'esistenza che mette in valore l'importanza della solidità e della corporeitä.

Quest'ultima tendenza è stata messa in evidenza da due critici, Giglioli e Tirinanzi De Medici. Tirinanzi De Medici osserva una forte presenza dell'esibizione di stati emozionali accompagnati da una componente corporale: se l'emotività si rivela un veicolo per superare l'anestetizzazione creata dalla pioggia di informazioni e pertanto uno strumento letterario per resistere ed esibire l'alterità dello specifico letterario rispetto alla comunicazione di massa, il corpo - più in generale il riferimento alla fisicità dell'esistenza - diviene superficie metaforizzante sulla quale si condensano la metafora, il simbolico e lo spirituale ${ }^{4}$. Anche Giglioli nota l'accompagnarsi nel romanzo italiano della componente fisica con quella emotiva, dove l'emotività pare discendere dalla descrizione di elementi corporali ("allora si sente che lo scrittore si rimbocca le maniche: la temperatura emotiva si alza, lo stile si impenna, la descrizione si trasforma in performance") $)^{5}$. Secondo Giglioli l'esibizione di stati emotivi e fisici, peraltro riconducibili sempre al basso ("proliferano qui il particolare sordido, la deformità, il grigiore, la sporcizia") ${ }^{6}$, si prestano da un lato a rendere credibili la descrizione della realtà quotidiana e dall'altro, nel loro tendere all'estremo sino al trionfo della morte e del corpo martoriato "oscenamente esposto allo sguardo", a squarciare il velo e porre fine alla menzogna del reale.

2. Simonetti Gianluigi, La letteratura circostante. Narrativa e poesia nell'Italia contemporanea, Bologna, Il Mulino, 2018, p. 24.

3. Tirinanzi De Medici Carlo, Il romanzo italiano contemporaneo. Dalla fine degli anni Settanta a oggi, Roma, Carocci, 2018, p. 197.

4. Ibid., p. 198.

5. Giglioli Daniele, Senza trauma Scritture dell'estremo e narrativa del nuovo millennio, Macerata, Quodlibet, 2011, p. 36.

6. Ibid.

7. Ibid. 
In effetti, soffermandoci unicamente sui tempi più recenti (gli ultimi dieci anni) si può notare come un numero significativo di opere di autori molto diversi tra loro presentino a diverse intensità l'affiorare della dimensione fisica e corporale, il che avviene per fini differenti: dall'indagine introspettiva del soggetto letterario alla metafora sociale. Senza alcuna pretesa ne volontà di presentare una lista esauriente, si possono tuttavia citare alcuni esempi: Il tempo materiale (2008) di Giorgio Vasta, Il contagio (2008) di Walter Siti, Italia de profundis (2008) di Giuseppe Genna, Quando verrai (2008) di Laura Pugno, L'ubicazione del bene (2009) di Giorgio Falco, A nome tuo (2011) di Mauro Covacich, Il peso della grazia (2012) di Christian Raimo, La vita in tempo di pace (2013) di Francesco Pecoraro, L'amore normale (2014) di Alessandra Sarchi, La ferocia (2014) di Nicola Lagioia.

Nel presente contributo si è deciso di non attraversare in maniera trasversale e generale tale tendenza ma di restringere il campo focale a tre autori: Nicola Lagioia, Giorgio Vasta e Giorgio Falco. Questa scelta muove principalmente da tre ragioni: le affinità biografiche dei tre autori, le quali si pongono infatti in un interessante e problematico dialogo con le tendenze della recente narrativa; le differenze strutturali notevoli tra le loro opere; le modalità simili del trattamento della corporeità. Nati tra il 1967 e il 1973, Lagioia, Vasta e Falco non partecipano in prima persona alla mutazione antropologica avvenuta tra gli anni Sessanta e Settanta, nascono e vivono già all'interno del solco del cambiamento sociale, economico, umano e politico che essa produce: il graduale disimpegno e il ritorno alla dimensione privata, il trionfo dei sistemi di comunicazione di massa e quindi dell'espansione dell'immaginario che riduce in parte la realtà a simulacro e feticcio, sino all'anestetizzazione emotiva poco sopra descritta. Per ogni autore si è scelto di analizzare le opere più emblematiche della loro produzione: La ferocia di Nicola Lagioia, Il tempo materiale di Giorgio Vasta e L'ubicazione del bene di Giorgio Falco.

Le tre opere mostrano importanti e fondamentali differenze: La ferocia è un novel pervaso da forti tendenze di mimesi realistiche; Il tempo materiale è un'opera surrealistica e allucinata costruita interamente su un impianto simbolico volto a ricostruire l'immaginario di un'epoca, quella degli anni di piombo ${ }^{8}$; L'ubicazione del bene è una raccolta di racconti brevi con echi provenienti dal minimalismo e l'iperrealismo americano. Cionondimeno, a fronte di queste profonde differenze, è stato possibile rinvenire due modalità raffigurative della corporeità che accomunano le tre opere: la presenza importante del bestiario, la rappresentazione

8. Donnarumma Raffaele, "Giorgio Vasta - Il tempo materiale", Allegoria, n. 60, 2009 , p. 222. 
del corpo martoriato e sofferente o del corpo abnorme e deforme. Per questioni di spazio, il breve studio qui proposto attraverserà solo una di queste due microtematiche, la rappresentazione del corpo. Al fine di evitare il catalogo, peraltro estremamente conciso e simile quindi ad una lista di occorrenze, ho deciso di attraversare le opere osservando in Vasta e Lagioia un passaggio particolarmente significativo, capace di mettere in prospettiva la tematica del corpo all'interno di tutto romanzo, mentre per Falco ho considerato un unico racconto capace di esemplificare parzialmente il trattamento del corpo fatto dall'autore. Ciò verrà effettuato in un procedere a intensità discendente: si inizierà pertanto da Il tempo materiale, dove i riferimenti al corpo sono estremamente insistiti e dove la scrittura è particolarmente materica, per giungere infine a L'ubicazione del bene, dove invece l'autore gioca su una scrittura basata sul distacco emotivo e su una presenza indiziaria del corpo.

\section{IL TEMPO MATERIALE: IL CORPO LINGUAGGIO E IL CORPO CHE ESONDA}

Il tempo materiale, come si diceva poco sopra seguendo la pista interpretativa di Donnarumma, è un romanzo che mette in scena l'immaginario di un'epoca: la fine degli anni '70 italiani stretti tra la definitiva vittoria del sistema mass mediatico e l'inasprimento della violenza politica. In questo contesto un gruppo di preadolescenti palermitani imita le gesta e il linguaggio dei brigatisti macchiandosi di diversi crimini in un crescendo di violenza che trova il culmine nel sequestro e omicidio del compagno di classe Morana, che richiama già nel nome Aldo Moro. Tuttavia, proprio come per le Br, inizia anche per loro la parabola discendente con le defezioni e le denunce interne al gruppo: al termine del romanzo Nimbo, uno dei ragazzini terroristi, si rifiuta di eliminare la compagna di scuola sordomuta Wimbow e denuncia i compagni. Ideologia e linguaggio, spinti all'orizzonte delle loro possibilità, fagocitano quindi i corpi e la vita per ridurre tutto a calcolo e purezza geometrica. Si tenterà di osservare rapidamente questo processo attraverso l'omicidio di Morana e di svelare le vie di fuga messe in pratica dal corpo.

Nel Tempo materiale i tre preadolescenti terroristi decidono di creare un linguaggio corporale chiamato alfamuto. Il linguaggio viene creato a partire dall'immaginario mass mediatico: le movenze di Celentano, le copertine dei dischi di Rino Gaetano, le pose di Raimondo Vianello o di Raffaella Carrà. L'ideologia si appropria in questo modo dell'immaginario popolare televisivo e cinematografico, ne svuota il contenuto e ne usa l'aspetto esteriore per fagocitarlo ("Noi prendiamo una forma famosa, fuori la lasciamo così com'è ma ne modifichiamo 
il contenuto $[. .$.$] prendiamo le posture idiote che tutti conoscono e le facciamo$ diventare messaggi in codice" "). Ciò avviene in una tensione quasi religiosa poiché la parola ideologica si fa carne, come il verbo divino, e allo stesso tempo piega il corpo al simbolico e all'astrazione come in un percorso di ascesi. Il processo di incarnazione dell'ideologia giunge al parossismo nell'omicidio di Morana: i tre giovani terroristi eseguono sul corpo di Morana pressioni e strette, il corpo viene deformato all'interno dell'ideologia difatti la vittima è sottoposta ad un interrogatorio corporale ("Lo interroghiamo con il corpo"10). Tuttavia la risposta è la parola vuota e svela pertanto il nucleo disabitato del linguaggio ideologico ("Ma cosa deve dirci? Niente" "guardo Morana negli occhi: dentro c'è solo una paura analfabeta" $\left.{ }^{\prime 1}\right)$. Le pagine che descrivono la tortura e la morte di Morana si succedono in un progressivo incremento della violenza dove il linguaggio corporale si fa sempre più esasperato in un aumentare della temperatura emotiva come descritto da Giglioli ("gli facciamo assumere altre posture, sempre esasperandole"12). Il sequestro e le torture durano una settimana, in quel lasso di tempo il corpo di Morana decade rapidamente ("Morana è sempre più magro"; "le croste si sono moltiplicate"13) e il susseguirsi delle torture porta a conseguenze mano a mano più gravi, Morana prima vomita e poi piange ("dal corpo di Morana vengono fuori dei singulti, degli scuotimenti sempre più forti' $^{\prime \prime 14}$ ), sino a giungere al drammatico epilogo: egli viene avvolto in una coperta e soffocato. Tale tracciato conduce all'esplosione finale che coincide con la morte di Morana e porta alla rivelazione: "colpire al cuore è una frase" perché "il cuore non c'è"15 dice il Nimbo. Nella morte di Morana si dischiude pertanto il vuoto della lotta, la sua sterilità e la sua riduzione ad estetica. Nel momento stesso in cui essa ingloba per vendetta l'immaginario mass mediatico e ne utilizza gli strumenti (i tre preadolescenti fotografano con una Polaroid Morana torturato imitando, tra l'altro, le gesta delle $\mathrm{Br}$ ) ne viene essa stessa fagocitata: "colpire al cuore è solo una frase" perché il "colpire al cuore" è solo uno slogan ad effetto che si esaurisce e mostra il fianco nel momento stesso in cui viene reso azione. La lotta dei tre ragazzi non è la distinzione e la divisione che crea la vita ma essa

9. Vasta Giorgio, Il tempo materiale, Roma, Minimum Fax, 2008, p. 113.

10. Ibid., p. 220.

11. Ibid., p. 226.

12. Ibid., p. 221.

13. Ibid., p. 222.

14. Ibid., p. 225.

15. Ibid., p. 227. 
crea e cova un morto: "lo partoriamo: il corpo morto di Morana viene fuori dai nostri corpi vivi" 16 .

$\mathrm{Al}$ termine del capitolo Nimbo ribadisce ancora una volta che "i corpi sono simboli", "i corpi sono corpi" ${ }^{17}$ risponde tuttavia il fratello Cotone nel loro dialogo immaginario. Infatti i corpi per Nimbo e i compagni devono essere inseriti nelle maglie rigide del simbolo privo del caso, nella rete geometrica pura e religiosamente disciplinata ("abbiamo passato un mese a eliminare il caso e a chiudere il mondo in una geometria perfetta. Ammetterlo, adesso, significa arrendersi" $\left.{ }^{\prime 18}\right)$. Così che, dopo la morte di Morana e in un procedere estremamente coerente delle decisioni dei personaggi del romanzo, la scelta della clandestinità diviene scelta di vita monastica in un processo di ideale ascesi religiosa volta alla soppressione della dimensione materica, corporale e umana ("La geometria e l'ossessione. L'estinzione dell'umano. La trasformazione di ogni centimetro di carne in disciplina. E quindi, adesso, la smaterializzazione della carne. La clandestinità come ultima forma di vita") ${ }^{19}$. Tuttavia la lotta e il sesso di questi corpi costretti sono appunto sterili. La fecondità si rende possibile solo quando al termine del romanzo i corpi finalmente esondano e mostrano il loro carattere irriducibile, quando Nimbo di fronte a Wimbow scopre che la necessità di elevare "il proprio amore a organismo" ${ }^{20}$. L'elevazione e l'ascesi si compiono quindi nell'“amare un corpo che è prima di tutto un organismo"21 perché il corpo appunto preesiste alla sua riduzione a pensiero e la sua bellezza si trova nel movimento non costretto. Wimbow, sorda e muta, è l'unica in grado di mostrare a Nimbo l'insufficienza della parola ("tu sei dove la frase cede e si sfalda [...] fino al vuoto") ${ }^{22}$. Nel momento in cui questa nuova verità si rivela a Nimbo egli si ritroverà presto a fare "l'eversore dell'eversione" 23 e nuove immagini di fecondità inizieranno ad apparirgli dove i corpi sono trasformazione, mutamento e movimento ("la materia si converte e noi con lei [...] e noi esistiamo ancora nel movimento e nella scrittura") ${ }^{24}$. Una visione cosmogonica di Nimbo chiude il romanzo e la consapevolezza che "alla fine delle parole

16. Ibid., p. 227.

17. Ibid., p. 230.

18. Ibid., p. 114.

19. Ibid., p. 233.

20. Ibid., p. 246.

21. Ibid.

22. Ibid., p. 271.

23. Ibid., p. 265.

24. Ibid., p. 271. 
comincia il pianto" 25 , ovvero inizia ciò che non ci è più concesso di pronunciare e si riprende il balbettio materico e primordiale, l'indicibile che si fa materia perché lo preesiste e lo oltrepassa.

\section{LA FEROCIA: IL CORPO SOVVERSIVO}

Il romanzo di Lagioia mette in scena una storia familiare nella Puglia degli ultimi decenni intrecciando ad essa le dinamiche sociali ed economiche. Il romanzo infatti descrive il crollo economico della famiglia Salvemini, del quale sono responsabili lo scoperchiamento dei loro illeciti economici e il disvelamento dei rapporti di potere che li sottendono. Lagioia costruisce tale narrazione seguendo il filo dell'inchiesta del noir: Michele Salvemini indaga sulla morte della sorella Clara e svela lentamente la fitta rete di rapporti di potere che coinvolge anche il padre Vittorio. Tuttavia il romanzo non narra unicamente il crollo economico della famiglia ma anche quello biologico: dalla morte violenta della figlia prediletta Clara sino alla morte per malattia di Vittorio. Tale procedere parallelo di tematiche biologiche ed economiche crea un intrecciarsi tra le due fitto e coerente. Si vedrà in questa sede come le prime si rivelino l'elemento capace di sovvertire gli equilibri delle seconde.

Un corpo in particolare nel romanzo di Lagioia si impone all'attenzione del lettore, quello di Clara. Clara muore in apertura del romanzo: vittima di un'orgia sadomasochista, vaga a piedi nella notte nella periferia barese e viene uccisa in un incidente stradale. La sua morte innesca l'indagine del fratello Michele circa le cause: egli scoprirà così le attività edili illecite del padre Vittorio che deciderà di denunciare provocando in questo modo la distruzione del suo impero economico. Per attraversare rapidamente la funzione del corpo di Clara nel romanzo ho ritenuto interessante soffermarmi in particolare su un passaggio dell'opera di Lagioia. Tale passaggio si rivela particolarmente significativo per due ragioni: vi è un cortocircuito tra gli interessi economici di Vittorio e il corpo di Clara; situato all'inizio del romanzo, sembra farsi carico di un valore epifanico in grado di illuminare il resto dell'opera.

Nell'estratto preso in esame vediamo Vittorio affacciarsi alla finestra della propria dimora e colpito da una gradevole brezza primaverile riflette sul proprio impero economico che si estende in ogni punto del globo, quasi perennemente illuminato dalla luce solare ("il tratto tra le dieci e le undici di sera [...] Era l'unico momento durante il quale la macchina del suo impero sarebbe stata ferma

25. Ibid., p. 274. 
ovunque" ${ }^{26}$. Tuttavia all'interno delle euforiche riflessioni sul proprio sistema imprenditoriale si insinuano due diverse inquietudini: una di carattere economico riguardo ad un progetto edilizio a Porto Allegro ("Non aveva ricevuto a quell'ora la notizia dei pasticci con il complesso turistico di Porto Allegro?") ${ }^{27}$; una di carattere biologico, Vittorio si interroga sul destino della figlia Clara ("Mentre aspettava di conoscere il destino di Clara") ${ }^{28}$ e sulla propria salute personale che sembra farsi più precaria ("E gli infarti?") ${ }^{29}$. Tali preoccupazioni, che insorgono come incisi nel flusso di pensiero, si impastano con quelle di carattere economico sino a fagocitarle. Infatti, se proseguiamo con la lettura del testo notiamo come Vittorio sposti in maniera abbastanza rapida il baricentro tematico delle proprie riflessioni: tralascia i pasticci di Porto Allegro e si concentra sui propri infarti, sulla sua ormai scarsa capacità polmonare, sulla memoria che inizia a divenire poco affidabile, e pone un parallelismo tra la sua mutazione corporale e la mutazione del mondo, ovvero il passaggio al capitalismo finanziario:

Eppure [...] Vittorio non poteva più fumare [...] e la memoria aveva smesso di essere quel prodigio che $i$ coetanei gli avevano invidiato a lungo. Per non parlare di come era cambiato il mondo. Avrebbe scommesso cento volte contro l'Argentina ma non avrebbe immaginato come pensieri, sfoghi e confidenze di milioni di adolescenti $[\ldots]$ potessero gonfiare il portafogli del più furbo di $\operatorname{loro}^{30}$.

Tuttavia la parentesi finanziaria si chiude rapidamente, distratta prima dall'arrivo di una coccinella all'interno della stanza e poi dal ritorno delle riflessioni su Clara. Per Vittorio la figlia è insondabile e sfuggente ("Di Clara aveva la sensazione di non capire mai abbastanza") ${ }^{31}$ e il suo aspetto ci appare per vivide zoomate su dettagli del corpo: i capelli rilasciati sulle spalle, le curve morbide, la bella bocca. Tale corporalità sminuzzata, simile alle descrizioni rapide e intense del linguaggio del videoclip, si innesta su un corpo evanescente che attraversa la dimora familiare come un soffio ("La ragazza passava leggera tra le stanze della villa. Difficile sentirla strepitare o anche solo provare a litigarci") ${ }^{32}$. È in questa

26. Lagioia Nicola, La ferocia, Torino, Einaudi, 2014, p. 30.

27. Ibid.

28. Ibid.

29. Ibid.

30. Ibid.

31. Ibid., p. 31.

32. Ibid., p. 32. 
tensione tra assenza e fulgida presenza che si crea l'insondabile di Clara, su questo solco agli occhi di Vittorio ella si fa idolo maya "il cui tocco scatena visioni dal futuro", e il futuro sono "le caravelle di Colombo, gli stupri di massa dei conquistatori" ${ }^{\prime 3}$. Seguendo tale traccia il passo qui analizzato ospita il senso profondo del personaggio di Clara e si fa profezia della fine: Clara è assenza e corpo vivido che attraversa tutto il romanzo, vive solo per lasciare lo spazio per descrivere la propria morte e poi compare a intermittenze regolari con una potenza corporale che si racchiude tutta nella continua descrizione del macrodettaglio, come se un occhio ossessivo la seguisse: dalle ecchimosi all'avvenente bellezza. In questa tensione si scatena la scossa tellurica che farà rovinare del tutto gli equilibri del romanzo e che si concluderà con la fine dell'impero economico dei Salvemini: l'assenza e la vivida presenza del corpo scatenante la pulsione erotica (la quale sarà causa della distruzione stessa del corpo di Clara) e l'amore del fratello Michele che dall'assenza e dalla distruzione muove le sue indagini e si fa caravella destinata a condurre alla propria fine l'antico impero paterno.

\section{L'UBiCAZIONE DEL BENE: IL CORPO OSCENO E IL REALE}

L'ubicazione del bene è una raccolta di racconti ambientati a Cortesforza, un immaginario comune dell'hinterland milanese. I racconti descrivono scene di vita quotidiana dove la predominante cromatica che delinea i tratti dei personaggi e del paesaggio è la scala di grigi: matrimoni che si dissolvono lentamente, rapporti umani ingabbiati nell'afasia, aspirazioni lavorative perennemente frustrate. Falco sceglie di rappresentare questo quadro attraverso una lingua piana e standard che si accompagna ad una narrazione scarna e concisa. Ciò lascia pochissimo spazio alle intrusioni emotive e nell'insieme la raccolta appare come un susseguirsi di fotografie dall'intento documentarista ${ }^{34}$. Anche la struttura interna dei singoli paragrafi procede per brevi sequenze intervallate dallo spazio bianco quasi a richiamare l'intervallo di muro bianco che separa le foto in una sala espositiva. All'interno di questo quadro stilistico, la corporeità e la fisicità hanno pertanto l'apparenza di reperti museali, oggetti esibiti senza coinvolgimento emotivo ma tuttavia capaci di far esplodere o illuminare le contraddizioni.

33. Ibid., p. 31 .

34. Tomasi Franco, "Immagini della megalopoli padana ne L'ubicazione del bene di Giorgio Falco", in РАРОтті Davide, TOMASI Franco (a cura di), La geografia del racconto. Sguardi interdisciplinari sul paesaggio urbano nella narrativa italiana contemporanea, Bruxelles, Peter Lang, 2014, p. 96. 
La lingua piana e grigia di Falco, le descrizioni distaccate e inaffettive, non impediscono una rappresentazione del corpo e degli stati corporali che richiama l'espressivismo e il grottesco, dove l'olfatto come spesso anche in Vasta e Lagioia $^{35}$ diviene elemento cognitivo privilegiato. All'interno della superficie levigata dei racconti tali descrizioni corporali assumono una valenza perturbante in grado di creare una crisi nel tessuto della narrazione e nella vita quotidiana di Cortesforza: il corpo si fa strumento di conoscenza. Il racconto omonimo al titolo della raccolta si rivela in questo senso particolarmente denso e interessante poiché possiamo rintracciarvi tali modalità rappresentative (l'olfatto; il corpo grottesco e abnorme) amalgamarsi tra loro in un impasto coerente.

Tutto il racconto ruota attorno ad una dialettica interno/esterno dove l'interno si caratterizza come una dimensione rassicurante e l'esterno come fonte di inquietudine; la tensione che provoca il ruotare di questi due elementi crea un'atmosfera elettrica e ansiosa che viene fatta esplodere al termine del racconto. L'elemento che permette tale esplosione è la rivelazione dell'orrore che si cela all'interno di Cortesforza, orrore incarnato dalla signora Giovanna. Nell'universo chiuso, conosciuto e familiare di Cortesforza abita infatti anche Giovanna, il cui corpo abnorme è percepito come un'oscenità ("i capelli color cenere, senza taglio, unti e appiccicati al cranio, si allungano aderenti fino alle tempie. Giovanna cammina spesso scalza sul marciapiede davanti casa [...]. I piedi sporchi mal custoditi dai sandali mezzi sfasciati, le parole impastate dalla saliva depositati agli angoli della bocca." $\left.{ }^{36}\right)$. La sua presenza a Cortesforza ci viene riferita poiché la signora Giovanna metterà in forno ancora vivo il proprio cane Scotty, provocando così un odore disgustoso che avvolgerà tutto il quartiere e richiamerà l'attenzione dei vicini e della polizia ("due sabati fa ho sentito un odore all'improvviso un odore fetido. Pensavo fosse un nuovo fertilizzante $[\ldots]$ si è alzato un putrido vento leggero [...] l'aria è diventata nauseabonda, come se nel 10/b si concentrasse il nucleo di morte a Cortesforza") ${ }^{37}$. L'olfatto ci conduce pertanto alla visione insopportabile del corpo di Giovanna e ci rivela il suo crimine ripugnante: la sua dimora è il nucleo di morte del quartiere, il Reale

35. In Il tempo materiale sono insistiti i richiami all'olfatto, per Nimbo l'odore è spesso materia. Ad esempio, l'odore di Corrado percepito attraverso lo schermo televisivo è l'odore "italiano e domenicale [...] quello che si genera sotto i vestiti indossati dal mattino presto". In Lagioia esso appare più raramente ma con grande forza espressionista, come vivide pennellate.

36. Falco Giorgio, L'ubicazione del bene, Torino, Einaudi, 2009, p. 40.

37. Ibid. p. 39. 
che fuoriesce a Cortesforza. Tuttavia una pista di presagi sembra preannunciare lungo il racconto tale orrore: prima con la larva viola che intacca il corpo sano del cavolfiore ${ }^{38}$ della cena di famiglia e poi con gli insistiti richiami alle facoltà cognitive dell'olfatto.

Seguendo tale pista di indizi possiamo rinvenire nel racconto, prima dell'odore proveniente dalla casa di Giovanna che rappresenta la conflagrazione finale, tre altri riferimenti all'odorato come strumento cognitivo in grado di porre in crisi il rapporto di separazione tra interno ed esterno. Incontriamo prima il profumo alla fragola sintetica dell'auto della voce narrante del racconto: a seguito di una lite familiare si chiude in auto e il profumo che vi trova provoca una sensazione di anestetizzazione ("mi sentivo un mutilato alla fragola" ${ }^{99}$ ) e pertanto di protezione dalle contraddizioni e dai conflitti della propria vita. Cionondimeno, se la percezione di sospensione e anestetizzazione sembra provocare una sensazione piacevole nel soggetto, essa si pone altresì in dialogo con la relazione matrimoniale mutilata e narcotizzata ("Alessandra dormiva sul divano $[. .$.$] Da quanto tempo non ci baciavamo?") { }^{40}$. Continuando sul nostro tracciato di segnali, qualche pagina dopo assistiamo ad una scena molto simile: l'odore degli interni del nuovo monovolume del vicino Gianni provoca nell'io della voce narrante una sensazione di stordimento ("Dentro il monovolume c'era una battaglia di odori [...] sono uscito dal monovolume stordito dal profumo" ${ }^{41}$ che sembra ancora una volta metterlo al riparo dal mondo esterno provocando un effetto narcotizzante ("l'aria dentro era fresca, sembrava impossibile che fuori potesse esserci un problema") ${ }^{42}$. Tuttavia questa serie di odori in grado di fornire requie e protezione viene interrotta da un primo odore sgradevole. Una domenica pomeriggio l'io narrante, la moglie Alessandra, i loro figli e la famiglia di Gianni si stanno dirigendo allo zoo safari con il monovolume e, nella calma annoiata delle code di auto e delle stazioni di servizio, un Tir parcheggia vicino al monovolume. Il Tir trasporta un carico di animali, agnelli e tori, e porta con sé un odore disgustoso di urina e feci che si insinua nel monovolume trovando una falla nel finestrino abbassato di Gianni ("cigolio di freni

38. Come si diceva all'inizio, nei tre autori è fortemente presente il bestiario. Si noterà qui rapidamente come la coccinella in Lagioia e la larva viola in Falco fungano da elementi perturbatori, interruzioni del flusso della normalità. Ribadendo una volta di più la capacità dirompente dell'elemento biologico.

39. Ibid., p. 23.

40. Ibid.

41. Ibid., p. 26.

42. Ibid., p. 27. 
e odore di stalla, merda e urina") ${ }^{43}$. Il camion e il suo carico di odori giungono a turbare la quiete quasi come un fantasma, un'irruzione improvvisa ("il camion sembrava arrivato da solo") ${ }^{44}$ e provocano una sensazione di inquietudine nelle due famiglie all'interno del monovolume ("Amore andiamo via [...] i camion non dovrebbero circolare la domenica") ${ }^{45}$. La reciproca autonomia di interno/ esterno viene pertanto turbata per la prima volta e l'odore sgradevole giunto dall'esterno si fa fautore di questo turbamento preannunciando il tragico epilogo e la rivelazione finale.

Corpo e olfatto si impastano nel racconto e l'olfatto si fa pista da seguire per giungere al corpo mostruoso che si cela al cuore della storia e di Cortesforza e che pone definitivamente in crisi il rapporto interno/esterno. Cortesforza e le sue bifamiliari non sono al riparo dall'osceno e dalla vita nel suo aspetto caotico e incontrollabile, i quali sono solo in apparenza condannate a rimanere all'esterno della nuova aerea residenziale.

\section{CONCLUSiOni}

L'analisi qui eseguita, pur senza alcuna pretesa di esaustività, ci mostra tuttavia una costante nelle tre opere: il corpo si caratterizza come prisma rivelatore, sovversione, strumento conoscitivo, scossa sul sismografo della narrazione. Il corpo nel momento della sua scoperta esonda e scombina gli equilibri della narrazione: in Giorgio Vasta la scoperta dell'irriducibilità del corpo a puro linguaggio e del suo preesistere ad esso conduce Nimbo a tradire i compagni; in Nicola Lagioia il corpo morto di Clara scatena la conflagrazione del sistema economico dei Salvemini; in Giorgio Falco il corpo abnorme irrompe e infrange l'ipocrita divisione tra interno ed esterno mostrando invece il rapporto dialettico ed osmotico tra le due dimensioni. Nelle tre opere, al pari del gesto di Fontana sulla tela, il corpo apre uno squarcio sul tessuto narrativo e ciò avviene perché il corpo antecede le altre verità e ne è la condizione di esistenza, il veicolo di conoscenza primaria.

Si può quindi giungere a una integrazione della proposta interpretativa di Tirinanzi e una conferma ulteriore della teoria di Giglioli: il corpo non è solo veicolo e strumento atto a superare l'anestetizzazione dei mass media, ma il suo affiorare si mostra talvolta come un processo ineluttabile. Infatti preesistendo alla pioggia mass mediatica esso è solo mascherato da quest'ultima: allorché il

\footnotetext{
43. Ibid., p. 29.

44. Ibid., p. 30.

45. Ibid.
} 
precipitare degli eventi ne causano il riaffiorare, esso infrange l'anestetizzazione del bombardamento mediatico, crea una ferita da cui iniziano ad affiorare le contraddizioni e provoca pertanto una crisi nel soggetto che ne esperisce la ricomparsa. In questo modo davvero gli stati emotivi e fisici squarciano il velo della realtà e ciò avviene poiché il corpo viene esperito come un fenomeno traumatico, come il Reale secondo l'interpretazione lacaniana che ne fa Žižek ${ }^{46}$. Il soggetto, disabituato al corpo e alla dimensione organica, vive la manifestazione corporale come irriducibile alla realtà e il suo affiorare si rivela uno shock che ne squaderna gli equilibri.

Matteo Ottaviano

Centre de Recherches Italiennes, CRIX, Université Paris Nanterre

46. ŽıžEK Slavoj, Benvenuti nel deserto del Reale. Cinque saggi sull'11 settembre e date simili, Milano, Meltemi, 2002. 
